\title{
Artroplastia reversa para as fraturas do úmero proximal: relato de caso
}

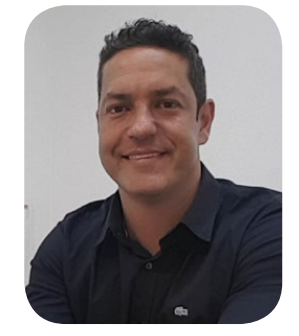

\author{
Roberto Barreto Maia1, Rogério Meira Barros ${ }^{1}$, Lucas Fraga Cunha da \\ Silva1, Marcus Vinícius Silva Santos ${ }^{1}$, David Corrêa ${ }^{1}$, Messias Neto'.
}

\section{INTRODUÇÃO}

As fraturas do úmero proximal (FUP) representam aproximadamente $5 \%$, quando consideramos todas as fraturas. São lesões frequentes em idosos após trauma de baixa energia, em decorrência da prevalência de osteoporose. Sua incidência tem aumentado nos últimos anos, elevação esta atribuída ao envelhecimento da população ${ }^{1}$. As principais complicações nos idosos são a lesão do manguito rotador (MR) e a osteonecrose da cabeça umeral, possíveis causas de dor e disfunção no ombro, perda da independência e, consequentemente, diminuição da qualidade de vida. Preservar a independência é o principal objetivo do tratamento proposto, considerando também o alívio da dor e a manutenção da função do ombro².

Para o tratamento, deve-se considerar a idade fisiológica do paciente, expectativa e demanda funcional, padrão e personalidade da fratura, qualidade $e$ estoque ósseo, instrumental técnico e experiência do cirurgião. Existem várias opções para o tratamento cirúrgico das fraturas do úmero proximal, tais como: fixação percutânea, placas bloqueadas, hastes intramedulares, hemiartroplastia (HA) e a artroplastia total reversa do ombro (ATR) ${ }^{3}$.

A ATR foi desenvolvida em 1985, por Grammont (Delta III), e, inicialmente, foi idealizada para tratamento da artropatia do manguito rotador. Este modelo de prótese tem como princípios biomecânicos a medialização e distalização do centro de rotação do ombro, tornando possível realizar a elevação do ombro mesmo na ausência dos tendões do manguito rotador. As indicações para o uso da ATR foram se expandindo rapidamente, devido ao sucesso alcançado no tratamento dos pacientes com a artropatia do manguito rotador. $\mathrm{E}$, atualmente, a prótese reversa tem sido utilizada também para o tratamento de fraturas complexas em idosos, àqueles que apresentam sinais radiográficos preditivos de isquemia da cabeça umeral e em pacientes com histórico pregresso de rupturas do $\mathrm{MR}^{2}$.

A utilização crescente da ATR em fraturas agudas do úmero proximal é justificada pelos bons resultados funcionais e menor taxa de complicações em comparação com a artroplastia parcial e a placa bloqueada ${ }^{4}$. A vantagem da ATR está na não exigência ou necessidade da consolidação dos tubérculos para se obter bons resultados 5 .

A melhor opção terapêutica para as FUP e os critérios para a utilização da ATR nas fraturas agudas ainda não estão totalmente esclarecidos. Desta maneira, relataremos um caso de uma paciente com diagnóstico de fratura do úmero proximal, submetida à ATR em nosso serviço ${ }^{4}$.

\section{RELATO DE CASO}

Paciente do sexo feminino, 79 anos, atendida em unidade de emergência no interior do estado, após queda da própria e queixa de dor em ombro direito. Após ter sido diagnosticada com fratura do úmero proximal, a paciente procurou o Hospital Santa Izabel para avaliação e conduta com especialista em ombro e cotovelo.

A paciente apresentava membro superior direito imobilizado em tipoia americana, equimose e hematomas no ombro e no braço direito. Não foram detectadas alterações neurovasculares. Após o exame físico foram realizadas radiografias e tomografia computadorizada do ombro e diagnosticado lesão complexa da extremidade proximal do úmero direito, classificada como uma fratura em 3 partes, de acordo Neer. Foi visualizado grande fragmento ósseo do tubérculo maior, medindo $5,11 \mathrm{~cm}$ (crânio-caudal), diástase entre os fragmentos principais e baixa qualidade óssea, bem como fatores preditivos para osteonecrose da cabeça umeral (figura 1). 


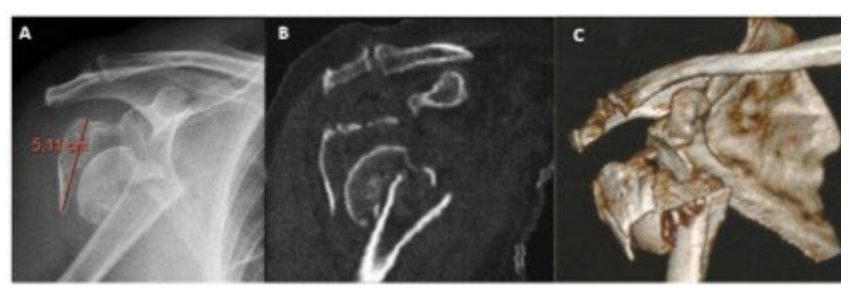

Figura 1 - Radiografia em frente do ombro (A), tomografia computadorizada com corte coronal $(B)$ e reconstrução 3D $(C)$ evidenciam fratura complexa do úmero proximal.

O procedimento cirúrgico foi efetuado sob anestesia geral e bloqueio de plexo braquial, em posição de cadeira de praia. Realizado acesso cirúrgico deltopeitoral, reparo dos tubérculos maior e menor, preparo da glenoide e fixação do componente glenoidal, inserção de haste umeral cimentada na diáfise, redução e amarria das tuberosidades tanto na porção proximal da haste quanto fixação na diáfise proximal do úmero. Em seguida, foi realizada a redução da prótese, testado estabilidade do implante, feito lavagem com solução fisiológica a 0,9\%, hemostasia, colocação de dreno suctor e fechamento de acesso cirúrgico com suturas por planos. Após curativo, o membro superior direito foi imobilizado em tipoia americana. As radiografias do ombro após o procedimento mostraram os implantes bem posicionados e redução adequada dos tubérculos (figura 2).

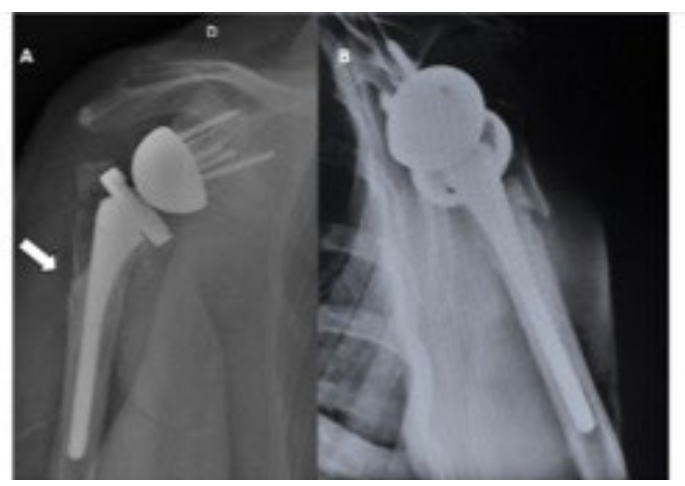

Figura 2 - Radiografia em frente do ombro $(A)$ e perfil escapular (B). Implante bem posicionado e com tubérculo maior em oposição à diáfise umeral (seta branca).

\section{DISCUSSÃO}

Estima-se que haja um aumento de três vezes na incidência das fraturas do úmero proximal até o ano de 2030 e que $70 \%$ destas ocorram em pessoas acima dos 60 anos $^{7}$. Embora alguns padrões de fratura sejam tratados muito mais objetivamente, a influência de fatores individuais (idade fisiológica, nível de atividade e independência de vida pregressa, expectativas com o tratamento e comorbidades) dificulta a uniformização e os protocolos de tratamento, tornando-o desafiador, mesmo para cirurgiões experientes ${ }^{3}$.

O tratamento cirúrgico com placa bloqueada apresenta bons a excelentes resultados funcionais em padrões de fraturas mais simples, já em lesões mais complexas (três ou quatro partes de Neer) e com baixa qualidade óssea, este tipo de abordagem apresenta taxa de complicações de aproximadamente $40 \%$, destacando-se o impacto subacromial, falha ou soltura do material de síntese, perda da redução, consolidação viciosa e osteonecrose ${ }^{7}$.

Para as fraturas com padrões complexos, fraturas-luxações, head split, pacientes com baixa qualidade óssea ou com sinais radiográficos preditivos de isquemia, a substituição articular utilizando a hemiartroplastia demonstra superioridade em relação às placas quanto ao alívio da dor, embora o resultado funcional possua grande desvio padrão e uma imprevisibilidade indesejável no desfecho, com relação ao resultado funcional ${ }^{8}$.

De maneira geral, alguns trabalhos relatam superioridade do score de Constant de 12 a 14 pontos em média para ART, em comparação com $H^{9,10}$. Uma revisão sistemática, realizada por Longo et. al., com 10 trabalhos, 256 pacientes e com follow-up médio de $27,8+21,8$ meses, concluiu que a ATR restaura função e promove alívio da dor nos pacientes com fratura do úmero proximal ${ }^{10}$. Outro demonstra a superioridade da ATR para a elevação anterior $\left(118^{\circ}\right.$ vs $\left.108^{\circ}\right)$ e menor rotação externa $\left(20^{\circ}\right.$ vs $\left.30^{\circ}\right)$, não constata diferença nos resultados funcionais; a artroplastia reversa total do ombro apresentou maior taxa de complicação $(9,6 \%$ vs $4,1 \%)$ e menor taxa de revisão $(0,93 \%$ vs $4,0 \%$ ), em comparação à $H A^{10}$.

A imprevisibilidade dos resultados funcionais com a $\mathrm{HA}$, a necessidade do bom posicionamento e a consolidação das tuberosidades para se obter bons resultados com este implante contribuem para a crescente utilização da ATR, nos pacientes idosos (acima dos 70 anos). Vários estudos advogam e defendem a utilização da artroplastia reversa para os pacientes com fraturas agudas que apresentem indicação de substituição articular, considerando uma melhor estimativa do resultado funcional ${ }^{6}$.

A ATR demonstrou ser uma opção terapêutica confiável e adequada para os pacientes com fraturas do 
úmero proximal, especialmente naqueles com padrões de fratura complexas em três ou quatro partes e em idosos com baixa qualidade óssea. Ressalta-se que é um procedimento cirúrgico que demanda técnica e experiência dos cirurgiões, e que apresenta taxas de complicações elevadas. A artroplastia reversa do ombro para tratamento de fraturas do úmero proximal proporciona alívio da dor e apresenta bons resultados funcionais.

\section{REFERÊNCIAS}

1. Cvetanovich GL, Frank RM, Chalmers PN, Verma NN, Nicholson GP, Romeo AA. Surgical Management of Proximal Humeral Fractures: The Emerging Role of Reverse Total Shoulder Arthroplasty. Orthopedics. 2016;39:e465-e473.

2. Wolfensperger $F$, Grüninger $P$, Dietrich $M$, Völlink $M$, Benninger E, Schläppi M, Meier C. Reverse shoulder arthroplasty for complex fractures of the proximal humerus in elderly patients: impact on the level of independency, early function, and pain medication. J Shoulder Elbow Surg. 2017 Aug;26(8):1462-1468.

3. Stanbury SJ, Voloshin I. Reverse shoulder arthroplasty for acute proximal humeral fractures in the geriatric patient: a review of the literature. Geriatr Orthop Surg Rehabil. 2011 Sep-Nov; 2(5-6): 181-186.

4. Chalmers PN, Keener JD. Expanding roles for reverse shoulder arthroplasty. Curr Rev Musculoskelet Med. 2016 Mar; 9(1): 40-48.

5. Acevedo DC, Vanbeek C, Lazarus MD, Williams GR, Abboud JA. Reverse shoulder arthroplasty for proximal humeral fractures: update on indications, technique, and results. J Shoulder Elbow Surg. 2014 Feb;23(2):279-89.

6. Jobin CM, Galdi B, Anakwenze OA, Ahmad CS, Levine WN. Reverse shoulder arthroplasty for the management of proximal humerus fractures. J Am Acad Orthop Surg. 2015 Mar;23(3):190-201.

7. Vijayvargiya M, Pathak A, Gaur S. Outcome analysis of locking plate fixation in proximal humerus fracture. J Clin Diagn Res. 2016 Aug; 10(8): RC01$\mathrm{RC} 05$.

8. Jones KJ, Dines DM, Gulotta L, Dines JS. Management of proximal humerus fractures utilizing reverse total shoulder arthroplasty. Curr Rev Musculoskelet Med. 2013 Mar;6(1):63-70.

9. Schairer WW, Nwachukwu BU, Lyman S, Craig EV, Gulotta LV. Reverse shoulder arthroplasty versus hemiarthroplasty for treatment of proximal humerus fractures. J Shoulder Elbow Surg. 2015 Oct;24(10):1560-6.
10. Longo UG, Petrillo S, Berton A, Denaro V. Reverse total shoulder arthroplasty for the management of fractures of the proximal humerus: a systematic review. Musculoskelet Surg. 2016 Aug;100(2):83-91.

1- Serviço de Ortopedia do HSI

Endereço para correspondência:

dcorreia@gmail.com 